\title{
Efeito da redução de ninhada sobre as respostas autonômicas e metabólicas de ratos Wistar
}

\author{
Effect of small litter size on the autonomic \\ and metabolic responses of Wistar rats
}

\author{
Wilson RINALDI ${ }^{1}$ \\ Tatiane Aparecida da Silva RIBEIRO ${ }^{1}$ \\ Angélica Sbrolini MARQUES ${ }^{1}$ \\ Gabriel Sérgio FABRICIO' \\ Laize Peron TÓFOLO' ${ }^{1}$ \\ Rodrigo Mello GOMES² \\ Fagner Cordeiro Vilar MENDES ${ }^{1}$ \\ Paulo Cezar de Freitas MATHIAS²
}

\section{RE S U M O}

\section{Objetivo}

Este estudo investigou o perfil lipídico e a atividade elétrica dos nervos parassimpático (vago superior) e simpático (localizado na região esplâncnica) de ratos obesos oriundos de ninhada reduzida.

\section{Métodos}

Foram pesquisados dois grupos distintos, com 12 animas cada um: ninhada padrão, padronizado em nove filhotes por ninhada, e ninhada reduzida, três filhotes por ninhada. O consumo de ração e peso corporal foi acompanhado do desmame até o final do protocolo experimental. Aos 90 dias de idade, os animais foram anestesiados com (Thiopental ${ }^{\circledR}$ ) e submetidos ao registro da atividade elétrica dos nervos simpático (vago) e parassimpático (da região esplâncnica); em seguida, foram sacrificados e retiradas e pesadas as gorduras retroperitoneal e periepididimal. Amostras de sangue foram coletadas para dosagens de glicemia, insulinemia, colesterol total, triglicerídeos e lipoproteína de alta densidade colesterol.

\section{Resultados}

Os ratos de ninhada reduzida apresentaram aumento da ingestão alimentar, peso corporal e tecido adiposo branco, quadros de hiperglicemia, hiperinsulinemia e hipercolesterolemia, aumento dos triglicérides e redução do lipoproteína de alta densidade colesterol.

\footnotetext{
1 Universidade Estadual de Maringá, Centro de Ciências Biológicas, Programa de Pós-Graduação em Educação Física. Av. Colombo, 5790, 87020-900, Maringá, PR, Brasil. Correspondência para/Correspondence to: W RINALDI. E-mail: <wrinaldi@uem.br>.

2 Universidade Estadual de Maringá, Centro de Ciências Biológicas, Departamento de Biologia Celular e Genética. Maringá, PR, Brasil.
} 
322 W RINALDI et al.

\section{Conclusão}

Quanto à atividade do nervo vago, os ratos ninhada reduzida apresentaram um aumento significativo em relação aos ratos ninhada padrão, e mesmo não havendo diferença na atividade simpática, o modelo ninhada reduzida mostrou-se eficaz para indução da obesidade, dislipidemia, hipercolesterolemia, hiperinsulinemia, hiperglicemia e desequilíbrio autonômico em roedores.

Termos de Indexação: Glicemia. Insulina. Obesidade. Sistema nervoso autonômico.

\section{A B S T R A C T}

\section{Objective}

This study investigated the lipid profile and electric activity of the parasympathetic (vagus nerve) and sympathetic (located in the splanchnic region) nerves of obese rats from small litters.

\section{Methods}

Two distinct groups were studied, each with 12 animals: normal litter with nine pups per litter and small litter, with three pups per litter. Chow intake and body weight were monitored from weaning until the end of the experimental protocol. At age 90 days, the animals were anesthetized with Thiopenta ${ }^{\circledR}$ for investigation of the electric activity of the sympathetic and parasympathetic nerves. They were then sacrificed for removal and weighing of the retroperitoneal and epididymal fat pads. Blood samples were collected for determination of blood glucose, insulin, total cholesterol, triglycerides and high-density lipoprotein cholesterol.

\section{Results}

The small litters rats had high food intake, body weight, white fat tissue, blood glucose, blood insulin, total cholesterol and triglycerides, and low high-density lipoprotein cholesterol levels.

\section{Conclusion}

The vagus nerve of the small litters rats was significantly more active than that of the normal litter rats. Sympathetic activity did not differ between the groups but the small litters model effectively promoted obesity, dyslipidemia, hypercholesterolemia, hyperinsulinemia, hyperglycemia and autonomic imbalance in rats.

Indexing terms: Blood glucose. Insulin. Obesity. Autonomic nervous system.

\section{N T R O D U ÇÃ O}

A obesidade está sendo considerada uma epidemia mundial, atingindo indivíduos de todas as faixas etárias e de diferentes níveis sociais, e acarretando um grande fator de risco para várias doenças degenerativas não-transmissíveis, como diabetes Mellitus tipo II, doenças cardiovasculares e hipertensão arterial $^{1-6}$. É frequente as alterações no perfil lipídico, com aumento nos níveis plasmáticos de Colesterol Total (CT), Triglicérides (TG), Lipoproteína de Baixa Densidade-colesterol (LDL-c) e diminuição nos níveis de Lipoproteína de Alta Densidade-colesterol (HDL-c), de pessoas que apresentam excesso de gordura corporal. As alterações nos níveis circulantes dessas biomoléculas podem levar ao desenvolvimento de doen- ças coronarianas, tanto em adultos quanto em crianças $^{7,8}$.

O ganho de peso, geralmente, tem relação direta com o aumento da ingestão energética, sendo a regulação do metabolismo realizada pelo Sistema Nervoso Central (SNC). O descontrole alimentar é capaz de afetar regiões cerebrais que podem modificar a regulação do peso corporal, principalmente as alterações na região hipotalâmica, que estão associadas a mudanças no comportamento alimentar e metabólico ${ }^{9}$. Algumas regiões específicas do hipotálamo têm por finalidade controlar a fome e a saciedade por meio da integração de sinais periféricos, incluindo a sinalização por hormônios gastrintestinais, leptina, insulina, citocinas, entre outros ${ }^{9}$. A partir desses sinais, são enviadas as aferências ao SNC, que envia à 
periferia as respostas necessárias para o controle da alimentação e da homeostase energética ${ }^{7,9}$.

Ainda no hipotálamo, mais especificamente no Núcleo Arqueado (ARC), encontram-se atividades importantes para o controle do metabolismo. Nesse núcleo, existem várias populações distintas de neurônios ${ }^{10}$ que produzem: a) o Neuropepitídeo Y (NPY) e o Agouti-related Peptide (AgRP), ambos neurotransmissores que agem em outras regiões do hipotálamo, promovendo aumento da ingestão de alimentos e ao mesmo tempo estimulando o armazenamento de nutrientes, e, dessa forma, fazendo a integração dos sinais anabólicos; b) a Pro-Opio-Melano-Cortin (POMC) e o Cocaine-and AmphetamineRegulated-Transcript (CART), os quais inibem o apetite e estimulam o gasto energético, integrando assim os sinais catabólicos ${ }^{10-12}$.

O controle alimentar e metabólico é realizado através do Sistema Nervoso Autônomo (SNA), subdividido em Sistema Nervoso Simpático (SNS) e Sistema Nervoso Parassimpático (SNP), com função predominantemente anabólica e catabólica, respectivamente ${ }^{11,13}$.

Estudos recentes sugerem que o SNS seja um dos mais importantes sistemas reguladores do balanço energético. Oscilações da atividade simpática podem afetar a quantidade de tecido adiposo em humanos, e a baixa atividade está presente em indivíduos adultos obesos ${ }^{10,13}$. Esses dados levam à hipótese de que alterações autonômicas devem originar a instalação da obesidade, ou pelo menos participar do seu desenvolvimento.

Além dos estudos epidemiológicos e clínicos, o uso de modelos experimentais de animais tem contribuído para o entendimento da fisiopatologia da obesidade. Esses modelos concentram-se, sobretudo, em roedores, principalmente ratos e camundongos, oriundos de linhagens selecionadas geneticamente e tratados com dietas ricas em gordura, excesso de alimentação e lesões no SNC ${ }^{16}$.

Um dos modelos experimentais de obesidade central, amplamente estudado, é obtido por meio da Redução de Ninhada (NR). Normalmente, as ratas parem em torno de Média $(M)=12$, Erro-Padrão Médio (EPM)=4 filhotes ${ }^{17,18}$ : padroniza-se a ninhada para 9 animais por rata. A redução de ninhada acontece no terceiro dia de vida, quando apenas três animais permanecem com a mãe. Essa redução promove uma programação metabólica que leva os filhotes à hiperalimentação. Após o desmame, aos 21 dias, mesmo sendo alimentados com ração balanceada com 18\%-24\% de proteína (ração comercial para roedores), eles têm maior consumo alimentar e ganham mais peso quando comparados aos filhotes oriundos de ninhadas normais. Esses animais na vida adulta apresentam hiperfagia, hiperinsulinemia, resistência periférica à insulina e obesidade ${ }^{19,20}$. Foi observado ainda que a redução da ninhada provoca alterações na estrutura e função de áreas hipotalâmicas, como, por exemplo, baixa resposta a estímulos anorexígenos no hipotálamo Ventromedial $(\mathrm{VMH})^{17-19}$. Sabe-se também que alterações nas estruturas hipotalâmicas quebram o equilíbrio autonômico, e lesões no VMH aumentam a atividade aferente do nervo vago subdiafragmático ${ }^{21,22}$.

O objetivo do presente trabalho foi verificar se a obesidade induzida por redução de ninhada modifica a atividade autonômica, o perfil lipídico e o controle glicêmico dos ratos adultos.

\section{M É T O D O S}

\section{Animais e modelo experimental}

Para este estudo, foram utilizados ratos Wistar, transferidos do Biotério Central da Universidade Estadual de Maringá para o Biotério Setorial do Laboratório de Biologia Celular da Secreção. Os animais foram submetidos a um período de cruzamento, na proporção de três fêmeas para cada macho. Ao constatar a prenhês, as ratas foram subdividas e acomodadas em caixas individuais. O número de filhotes foi padronizado com nove animais para Ninhada Padrão (NN) e três para $\mathrm{NR}^{23}$. O desmame foi realizado aos 21 dias $^{19}$ 
e os grupos foram padronizados na proporção de três animais para cada caixa (Figura 1).

O consumo de ração e o peso corporal foram medidos semanalmente, do desmame até os 91 dias de vida. Durante todo o período experimental, os animais permaneceram em condições controladas de luminosidade $(12 \mathrm{~h}$ claro $-12 \mathrm{~h}$ escuro) e temperatura constante $(M=22,0$, $E P M=2^{\circ} \mathrm{C}$ ), recebendo água e ração comercial (Nuvital-Curitiba) ad libitum.

\section{Glicemia e insulinemia de jejum}

Aos 91 dias de vida, os animais foram anestesiados com ketamina (3,0mg) e xilazina $(0,6 \mathrm{mg})$ para cada 100 gramas de massa corporal e submetidos a um processo cirúrgico de implantação de uma cânula de silicone na veia jugular direita. Após 12 horas de jejum, foi realizado o Teste de Intolerância à Glicose Intravenoso (ivGTT). Amostras de sangue $(0,200 \mathrm{~mL}-0,300 \mathrm{~mL})$ foram retiradas, via cânula. Após a retirada do tempo 0 , administrou-se uma carga de glicose $1 \mathrm{~g} / \mathrm{kg}$ de peso corporal. As amostras de sangue foram coletadas nos tempos 5, 15, 30 e 45 minutos. O sangue foi centrifugado (3000rpm por 10 minutos) e retirado o plasma para dosagem de glicose pela técnica da glicose-oxidade (Gold Analisa $\left.{ }^{\circledR}\right)^{24}$.

A insulina foi dosada através da técnica por Radioimunoensaio (RIA). Os resultados foram expressos em $\mathrm{mmol} / \mathrm{L}$ para glicemia e $\mathrm{ng} / \mathrm{mL}$ para insulinemia. Para avaliar a resistência à insulina periférica, foi utilizada a área sob a curva de toda insulinemia, e para o cálculo do índice insulino-

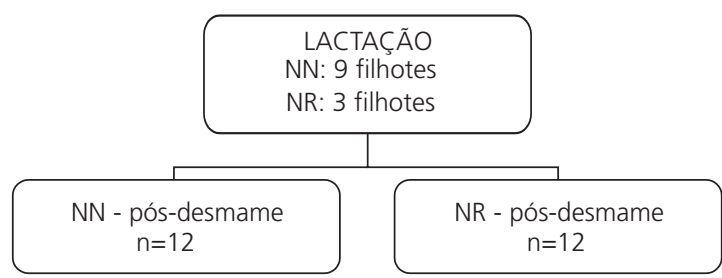

Figura 1. Fluxograma da padronização das ninhadas de animais de Ninhada Padão (NN) e Ninhada Reduzida (NR) no período da lactação e pós-desmame. gênico, utilizou-se a glicemia durante o ivGTT. O índice Homeostatic Model Assessment for Insulin Resistance (HOMA-IR) foi expresso pela equação: insulina basal (ng/mL) x glicose basal (mg/dL)/22,5.

\section{Perfil lipídico}

O plasma coletado em jejum de 12 horas foi utilizado para as dosagens do colesterol total, colesterol HDL-c e triglicérides, pelo método enzimático colorimétrico (Gold Analisa ${ }^{\circledR}$ ). Os resultados foram expressos em mg/dL ${ }^{24}$.

\section{Atividade do SNA}

SNP: Após 2 dias, os mesmos animais submetidos ao ivGTT de ambos os grupos foram anestesiados com tiopental sódico $(45 \mathrm{mg} / 1000 \mathrm{~g}$ de peso animal), sendo submetidos a uma incisão cirúrgica longitudinal na face anterior cervical para possibilitar a dissecação do ramo superior vagal direito. Após o nervo ser isolado, o animal foi colocado em uma gaiola de Faraday para evitar interferências eletromagnéticas externas que pudessem alterar os registros elétricos. Com o auxílio de um fio de algodão transpassado sob o nervo, o filamento neural isolado foi assentado sobre um par de eletrodos de prata $(0,6 \mathrm{~mm})$ e conectado a um sistema eletrônico que transformava o sinal elétrico em sinal sonoro. O sinal foi filtrado para excluir ruídos abaixo de $1 \mathrm{KHz}$ e acima de $80 \mathrm{KHz}$, e foi amplificado 10 mil vezes. Os registros foram convertidos para sistema digital e armazenados em arquivos eletrônicos (Insight ${ }^{\circledast}$, Ribeirão Preto, SP, Brasil) para posterior análise. Após dois minutos iniciais, o registro da atividade do nervo foi tomado por dez minutos. Aleatoriamente foi escolhido de cada registro/animal de 5 a 7 setores com 15 segundos para a contagem de espículas de $\mathrm{mV}$ que superavam o zero. Os resultados da atividade elétrica do nervo foram expressos em número de espículas por 5 segundos (spikes/5s) ${ }^{25}$.

SNS: Após encerrado o registro da atividade elétrica do nervo vago, com o animal ainda 
anestesiado, foi realizada uma laparotomia e localizado um ramo do nervo simpático localizado na região esplâncnica com origem no plexo lombar na região de $L 2$, que se estende até o tecido adiposo retroperitoneal; a dissecação e o registro da atividade elétrica do nervo foram realizados como descrito para o nervo vago.

\section{Avaliação da obesidade}

Foram aferidos o peso corporal em gramas e o Comprimento Nasoanal (CNA) em centímetros para calcular índice de Lee $\left[\right.$ Peso $(\mathrm{g})^{1 / 3} \div \mathrm{CNA}(\mathrm{cm})$ $x$ 1000], utilizado como estimativa de sobrepeso. As gorduras periepididimal e retroperitonial foram extraídas e pesadas para o fornecimento da estimativa do acúmulo de gordura tecidual do animal ${ }^{26}$. Todos os experimentos foram realizados de acordo com as normas do Colégio Brasileiro de Experimentação Animal (COBEA).

\section{Análise estatística dos dados}

Os resultados estão apresentados em M e EPM. Para a comparação das diferenças entre os grupos experimentais foi utilizado o teste $t$ de Student, com nível de significância estabelecido em $p<0,05$.

O estudo foi aprovado pelo Comitê de Conduta Ética no Uso de Animais em Experimentação da Universidade Estadual de Maringá, protocolo n 028/2009, em 01/09/2009.

\section{RES U LT A D OS}

\section{Obesidade e consumo de ração}

Os ratos NR apresentaram aumentos de 9,4\% no peso corporal, 4,1\% no índice de Lee, $140,6 \%$ e $88,6 \%$ nas gorduras periepididimal e retroperitoneal respectivamente quando comparados aos NN, $p<0,05$ (Tabela 1). Os ratos NR apresentaram aumentos de $14,5 \%$ no consumo de ração quando comparados aos animais de NN, $p<0,05$ (Figura 2).

\section{Intolerância à glicose e resistência à insulina}

A glicemia basal dos animais NR foi $31,7 \%$ e a insulinemia basal $40,8 \%$ maiores que dos animais NN, $p<0,05$ (Tabela 2). O índice HOMA-IR aumentou $85,1 \%$ nos ratos pertencentes ao NR quando comparado aos valores obtidos para os animais NN, $p<0,05$.

\section{Perfil lipídico}

Os animais NR apresentaram uma concentração plasmática de colesterol total 19,5\% maior que os animais NN $(p<0,05)$; a concentração plasmática de triglicérides foi $28,8 \%$ mais elevada que os animais NN, $p<0,05$. A concentração plasmática de HDL-c foi a que apresentou maior alteração, tendo os animais NR mostrado uma diminuição de 53,8\% em relação aos animais NN, $p<0,05$ (Figura 2).

Tabela 1. Efeito da redução de ninhada sobre o peso corporal, acúmulo de gordura e consumo de ração.

\begin{tabular}{|c|c|c|c|c|}
\hline & \multicolumn{2}{|c|}{ Ninhada Normal } & \multicolumn{2}{|c|}{ Ninhada Reduzida } \\
\hline & M & EPM & M & EPM \\
\hline Peso corporal (g) & 386,70 & 18,98 & 423,10 & $28,60^{*}$ \\
\hline Índice de Lee $\left(\mathrm{g}^{1 / 3} / \mathrm{cm}\right)$ & 311,50 & 1,60 & 324,20 & $1,60^{*}$ \\
\hline Gordura periepididimal (g/100g) & 0,64 & 0,02 & 1,54 & $0,10^{*}$ \\
\hline Gordura retroperitonial $(\mathrm{g} / 100 \mathrm{~g})$ & 0,88 & 0,18 & 1,66 & $0,27^{*}$ \\
\hline
\end{tabular}

Os valores representam a Media (M) e Erro-Padrão da Média (EPM). * $p<0,05$ na comparação dos valores do grupo Ninhada Reduzida com os do grupo Ninhada Padrão. 
326 | W RINALDI et al.

Tabela 2. Efeito da redução de ninhada sobre a glicose e a insulina basais, incremento da glicose e insulina durante o ivGTT, índice de resistência à insulina, índice insulinogênico e perfil lipídico.

\begin{tabular}{lccccc}
\hline & \multicolumn{2}{c}{ Ninhada Padrão } & & \multicolumn{2}{c}{ Ninhada Reduzida } \\
\cline { 2 - 3 } \cline { 5 - 6 } & M & ERP & & M & ERP \\
\hline Glicemia Basal (mmol/L) & 4,61 & 0,23 & & 6,07 & $0,25^{*}$ \\
Insulinemia Basal (ng/mL) & 0,49 & 0,04 & & 0,69 & $0,02^{*}$ \\
HOMA-IR & 1,81 & 0,007 & & 3,35 & $0,004^{*}$ \\
Colesterol Total (mg/dL) & 68,09 & 2,51 & & 81,36 & $5,7^{*}$ \\
HDL Colesterol (mg/dL) & 62,11 & 4,06 & & 28,71 & $2,26^{*}$ \\
Triglicérides (mg/dL) & 60,40 & 3,49 & & 77,79 & $9,7^{*}$ \\
\hline
\end{tabular}

Os valores representam a Media (M) e Erro-padrão da Média (EPM). ${ }^{*} p<0,05$ na comparação dos valores do grupo Ninhada Reduzida (NR) com os do grupo Ninhada Padrão (NN).

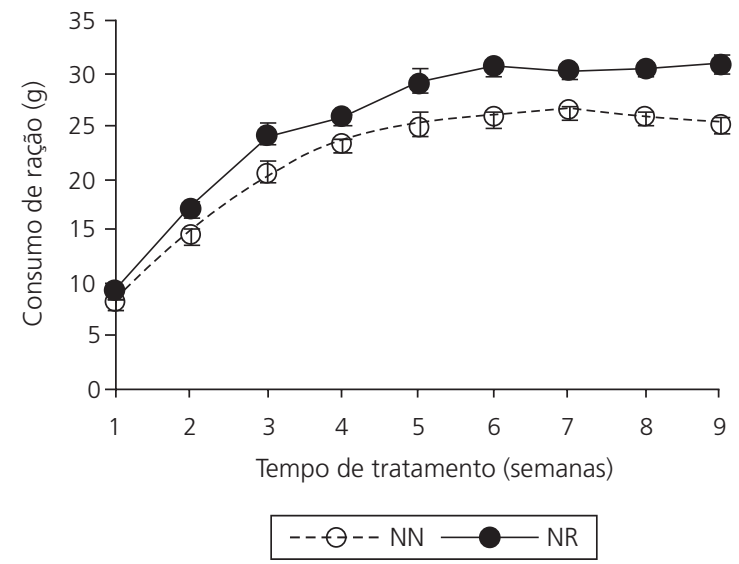

Figura 2. Comparação entre os valores do consumo alimentar de 10 semanas de animais da Ninhada Reduzida (NR) e da Ninhada Padrão (NN).

Nota: Teste $t$ de Student $(p<0,05)$.

\section{Atividade do SNA}

A quantidade de disparos elétricos do nervo vago de ratos NR foi $22,8 \%$ mais elevada que nos animais $N N, p<0,05$. Porém, a atividade elétrica do nervo simpático não apresentou qualquer diferença entre os dois grupos (Figura 3).

\section{I S C U S S Ã O}

Os resultados apontam que a redução do número de filhotes da prole durante o período lactacional foi eficaz para indução da obesidade em ratos Wistar. Conforme a literatura ${ }^{17,19,20}$, esses animais apresentam hiperfagia, ganho de peso e aumento do tecido adiposo. Plagemann et al.23,27 mostraram que animais NR apresentam hiperinsulinemia no período lactacional, perdurando até a vida adulta. Observou-se ainda que esses animais apresentavam hiperfagia, aumento do peso corporal e do tecido adiposo, alteração no perfil lipídico, hipoplasia e hipotrofia no Hipotálamo Ventro-Medial $(\mathrm{VMH})^{19}$.

O hipotálamo é uma das regiões centrais mais completas da citoarquitetura neural, ficando, durante o período lactacional, susceptível a influências metabólicas que podem alterar sua homeostase ${ }^{28,29}$. Dessa forma, caracteriza-se como uma importante janela de desenvolvimento metabólico. Estudos com ratas prenhes submetidas a dietas de alto teor energético resultaram em obesidade da prole na vida adulta, bem como hiperfagia e resistência à insulina.

A obesidade apresenta uma relação direta com a resistência à insulina e a intolerância à glicose, predispõe a diabetes Mellitus Tipo II (DMII) ${ }^{18,27}$ e está determinada por diversos fatores metabólicos neurais e endócrinos ${ }^{30}$. Estudos demonstram que alterações da sinalização de insulina no tecido adiposo estão associadas à intolerância à glicose, causada por decréscimo do GLUT-4, que é o principal transportador de glicose e depleção de GLUT-1 que também atua no transporte de glicose, estimulando através da insulina a lipogênese e a inibição da lipólise ${ }^{19}$. Efeito semelhante ocorre no tecido muscular em animais NR com decréscimo significativo do GLUT-4 ${ }^{19}$.

Plagemann et al.18,31 mostraram que animais expostos a uma superalimentação no 
A

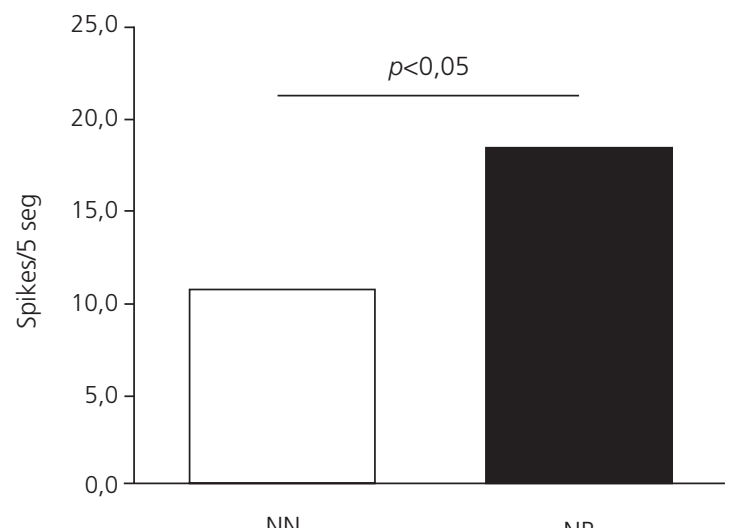

NN
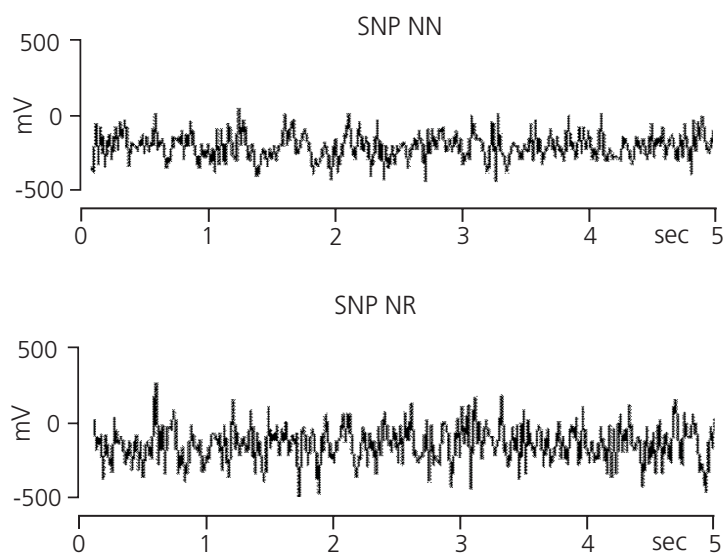

B

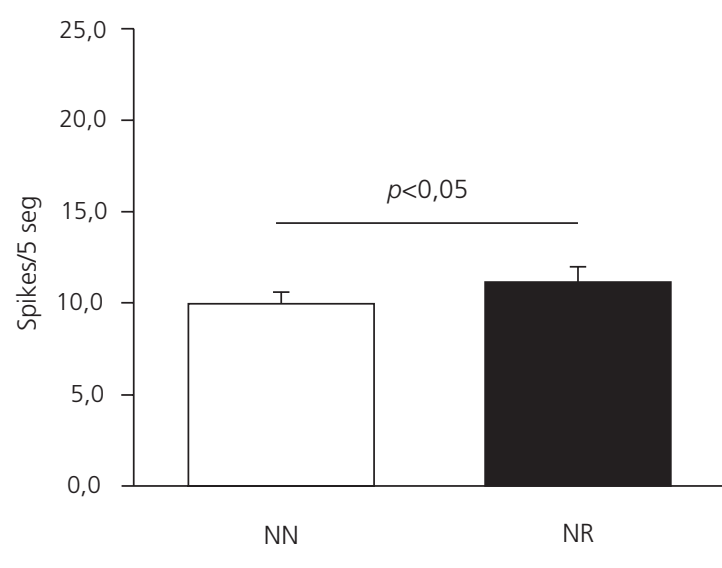

SNS NN

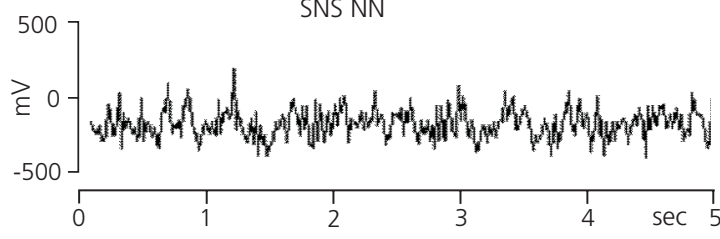

SNS NR

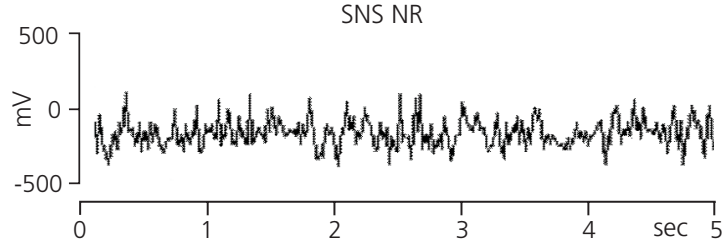

Figura 3. Efeito da redução de ninhada sobre a atividade elétrica do sistema nervoso parassimpático - nervo vago (A) e do sistema nervoso simpático - nervo retroperitoneal (B) em ratos NR e NN.

Nota: Teste $t$ de Student $(p<0,05)$; NR: Ninhada Reduzida; NN: Ninhada Padrão.

período pré-natal apresentaram hiperinsulinemia precoce (a partir do sétimo dia de vida) e aos 250 dias de idade apresentaram aumento na glicemia de jejum ${ }^{18}$. No presente estudo os animais NR apresentaram um quadro de hiperinsulinemia e hiperglicemia basal, corroborando estudos que evidenciaram que, na fase inicial da obesidade, a hiperinsulinemia é suficiente para manter a glicemia basal, no entanto, não evita a intolerância à glicose em fases tardias da vida18,27,28,31.

Níveis elevados de insulina plasmática em jejum são os primeiros sinais para o desenvolvimento do DMII. Em estágios iniciais, devido à resistência à insulina, as células $\beta$-pancreáticas aumentam a produção e a secreção de insulina como mecanismo compensatório, enquanto a tolerância à glicose permanece normal. Porém, com a evolução do quadro, observa-se um declínio na secreção de insulina e queda na tolerância à glicose. Portanto, o modelo RN foi eficaz na indução da obesidade e na resistência à insulina.

Alterações encontradas para os valores de colesterol total, triglicérides e HDL-c em animais NR apontam para um quadro de dislipidemia, que se relaciona à resultados da literatura ${ }^{32,33}$. Estudos apontam ainda que a obesidade está diretamente associada a comorbidades, como a dislipidemia ${ }^{5-7,20,32}$

O SNA tem papel fundamental no controle metabólico ${ }^{23,34}$. O equilíbrio do metabolismo corporal prescinde de ações hormonais que são coordenadas entre SNS e SNP ${ }^{10}$. Ao longo do desenvolvimento, surgem adaptações funcionais necessárias para garantir a manutenção desse controle e evitar alterações do metabolismo ${ }^{24,34,35}$. O hipotálamo de roedores encerra seu desen- 
volvimento no final do desmame, perdurando por algumas semanas, portanto qualquer evento metabólico adverso nesse período pode comprometer a organização estrutural do sistema nervoso central e determinar alterações funcionais importantes $^{18}$.

Verificou-se que em condições fisiológicas normais há um equilíbrio entre o SNS e SNP, com predomínio do SNS (anabólico) ${ }^{10}$. Nos resultados deste estudo, com animais NR, foi observado que a atividade simpática estava reduzida e a parassimpática encontrava-se aumentada, o que caracteriza o descontrole energético. Estudos demonstraram que a desregulação simpática e a parassimpática de animais obesos estão associadas a alterações hormonais e metabólicas, assim como foi observado em nossos resultados de glicemia, insulinêmica e perfil lipídico. O aumento da atividade simpática pode afetar a quantidade de massa adiposa em obesos, apresentando atividade simpática reduzida ${ }^{36}$.

O cérebro desempenha importante papel na homeostase energética ${ }^{37}$. O controle da ingestão de nutrientes e o equilíbrio homeostático dependem de sinais periféricos que atuam diretamente sobre SNC, desempenhando a região hipotalâmica um papel chave na integração de sinais periféricos e controle da homeostase energética $^{29}$. Circuitos neuronais desse sistema, principalmente aqueles localizados no hipotálamo, recebem e integram sinais metabólicos, endócrinos e neurais, coordenando respostas, através do metabolismo, com objetivo de controle do peso corporal ${ }^{38}$. Estudos de áreas hipotalâmicas demonstraram que o ARQ e o VMH são dois importantes núcleos reguladores da ingestão alimentar e do gasto energético, e podem ser afetados no modelo de $\mathrm{NR}^{18,19}$.

Animais desse modelo experimental apresentam quadros de hiperfagia, que podem estar relacionados com alguma disfunção do $\mathrm{VMH}$ e/ou ARC, pois o excesso de nutrientes pode ser responsável pelas alterações na densidade e atividade neural de núcleos hipotalâmicos dos animais NR ${ }^{17-19}$.

O Sistema Nervoso Central emite sinais aferentes e eferentes ao Sistema Nervoso Autô- nomo (SNA), que geram um equilíbrio entre SNC e a periferia ${ }^{39}$. No entanto, essa relação das vias de comunicação no controle da ingestão alimentar e do peso corporal é mediada principalmente pelo sistema nervoso autônomo ${ }^{39,12}$. Esses mecanismos são subdivididos em SNS e SNP: o primeiro estimula o catabolismo, e o segundo anabolismo, ambos controlados por áreas localizadas predominantemente no hipotálamo, associando a obesidade ao desequilíbrio da atividade do SNA ${ }^{33}$. A superalimentação durante a vida perinatal contribui para o desenvolvimento da obesidade, associada a distúrbios metabólicos e cardiovasculares na vida adulta ${ }^{22,31}$.

Animais NR do presente estudo apresentaram hiperinsulinemia de jejum acompanhada de hiperglicemia de repouso. Embora a atividade elétrica simpática do tecido adiposo retroperitoneal não tenha apresentado diferença significativa entre NN e NR, a hiperinsulinemia de jejum acompanhada por intolerância à glicose pode estar relacionada à ação do SNP. Os resultados mostram que o nervo vago em estado de repouso apresenta maior quantidade de disparos em ratos NR. Estudos indicam que a origem da obesidade é impulsionada por baixa atividade do SNS e alta atividade do SNP, representado pela hiperatividade vaga| ${ }^{21,40,41}$.

A estimulação do SNP promove o armazenamento dos substratos energéticos, tais como glicose e ácidos graxos livres, 4,36,39. O aumento da secreção de insulina está relacionado também ao aumento da atividade parassimpática. Isso acontece devido à maior liberação de acetilcolina nos terminais pancreáticos, fazendo com que ocorra diminuição da glicemia, pela estimulação da captação de glicose e supressão de sua produção. Em tecidos insulino-sensíveis, como o muscular e o adiposo, a insulina estimula a captação, estoque e a utilização de glicose, tendo assim um efeito anabólico, provocando armazenamento energético ${ }^{35}$.

\section{CONCLUSÃ O}

Os resultados demonstraram que animais expostos a uma superalimentação no período 
pré-natal NR apresentam aumento do consumo alimentar, do peso corporal, da gordura corporal, da hiperglicemia, da hiperinsulinemia, da dislipidemia e alterações na atividade autonômica. Observou-se um aumento da atividade parassimpática que pode ter contribuído com a hiperinsulemia, com um possível quadro de resistência à insulina e, por consequência, com um desequilíbrio da homeostasia glicêmica. A partir do exposto, faz-se necessário explorar esse modelo experimental, pois alterações no SNA causadas pela obesidade podem gerar distúrbios metabólicos, tais como diabetes e obesidade. Estes estudos poderão auxiliar na compreensão de mecanismos que possam atenuar essas alterações e, com isso, estabelecer mecanismos fisiopatológicos que abrirão novos caminhos profiláticos e/ou terapêuticos na prevenção e no combate da obesidade e de suas consequências.

\section{A GRADECIMENTOS}

À Coordenação de Aperfeiçoamento de Pessoal de Nível Superior (Capes) e ao Conselho Nacional de Desenvolvimento Científico e Tecnológico $(\mathrm{CNPq})$, pelo apoio financeiro. Não há conflito de interesse por parte dos autores e dos órgãos financiadores que possa interferir na publicação dos resultados deste trabalho.

\section{COLABORADORES}

W RINALDI, TAS RIBEIRO, LP TÓFOLO e GS FABRíCIO contribuíram em vários aspectos do projeto, da obtenção dos animais à retirada das amostras e análise dos resultados. FCV MENDES interpretou os dados e redigiu o artigo. RM GOMES realizou análise estatística. PCF MATHIAS realizou revisão crítica do artigo.

\section{REFERÊ N CIAS}

1. Farmer SR. Molecular determinants of brown adipocyte formation and function. Genes Dev. 2008; 22(10):1269-75.

2. Berthoud HR. Homeostatic and non-homeostatic pathways involved in the control of food intake and energy balance. Obesity. 2006; 14(Suppl 5): S197-S200.

3. Pagano C, Marzolo M, Granzotto M, Ricquier D, Federspil G, Vettor R. Acute effects of exercise on circulating leptin in lean and genetically obese fa/ fa rats. Biochem Biophys Res Commun. 1999; 255(3):698-702.

4. Yan ZC, Liu DY, Zhang LL, Shen CY, Ma QL, Cao $T B$, et al. Exercise reduces adipose tissue via cannabinoid receptor type 1 which is regulated by peroxisome proliferator-activated receptor-delta. Biochem Biophys Res Commun. 2007; 354(2): 427-33.

5. Wisse BE, Kim F, Schwartz MW. Physiology. An integrative view of obesity. Science. 2007; 318 (5852):928-9.

6. Waki H, Tontonoz P. Endocrine functions of adipose tissue. Annu Rev Pathol. 2007; 2:31-56.

7. Lima SCV, Arraes RF, Almeida MG, Souza ZM, Pedrosa LFC. Perfil lipídico e peroxidação de lípidios no plasma em crianças e adolecentes com sobrepeso e obesidade. J Pediatr. 2004; 80:23-28.

8. Lunardi CC, Petroski EL. Body mass index, waist circumference and skinfolds for predicting lipid abnormalities in 11 years old children. Arq Bras Endocrinol Metabol. 2008; 52(6):1009-14.

9. Blundell JE. Perspective on the central control of appetite. Obesity. 2006; 14(Suppl 4):S160-S3.

10. Grill HJ. Distributed neural control of energy balance: contributions from hindbrain and hypothalamus. Obesity. 2006; 14(Suppl 5): S216- S21.

11. Young JB. Developmental origins of obesity: a sympathoadrenal perspective. Int J Obes (London). 2006; 30(Suppl 4):S41-S9.

12. German JP, Thaler JP, Wisse BE, Oh IS, Sarruf DA, Matsen ME, et al. Leptin activates a novel CNS mechanism for insulin-independent normalization of severe diabetic hyperglycemia. Endocrinology. 2011; 152(2):394-404.

13. Teff KL. Visceral nerves: vagal and sympathetic innervation. JPEN J Parenter Enteral Nutr. 2008; 32(5):569-71.

14. Benoit SC, Clegg DJ, Seeley RJ, Woods SC. Insulin and leptin as adiposity signals. Recent Prog Horm Res. 2004; 59:267-85.

15. Campos KE, Sinzato YK, Pimenta WP, Rudge MV, Damasceno DC. Effect of maternal obesity on diabetes development in adult rat offspring. Life Sci. 2007; 81(19-20):1473-8.

16. Bray GA, Paeratakul S, Popkin BM. Dietary fat and obesity: a review of animal, clinical and epidemiological studies. Physiol Behav. 2004; 83(4):549-55. 
17. Plagemann A, Harder T, Rake A, Janert $U$, Melchior $\mathrm{K}$, Rohde W, et al. Morphological alterations of hypothalamic nuclei due to intrahypothalamic hyperinsulinism in newborn rats. Int J Dev Neurosci. 1999; 17(1):37-44.

18. Plagemann A, Harder T, Rake A, Voits M, Fink $H$, Rohde W, et al. Perinatal elevation of hypothalamic insulin, acquired malformation of hypothalamic galaninergic neurons, and syndrome $x$-like alterations in adulthood of neonatally overfed rats. Brain Res. 1999; 836(1-2):146-55.

19. Rodrigues $A L$, Souza EP, Silva SV, Rodrigues DS, Nascimento $A B$, Barja-Fidalgo $C$, et al. Low expression of insulin signaling molecules impairs glucose uptake in adipocytes after early overnutrition. J Endocrinol. 2007; 195(3):485-94.

20. Rinaldi W, Gomes RM, Dias MJ, Marques AS, Scomparin DX, Martins AG, et al. O rompimento da homeostase glicêmica em ratos obesos oriundos de ninhadas reduzidas. Endocrinol Diabetes Clin Exp. 2008; 8(4):911-15.

21. Kiba T, Tanaka K, Inoue S, Endo O, Takamura Y. Comparison of DNA contents of visceral organs in rats with ventromedial hypothalamic lesions and fed a high fat diet. Neurosci Lett. 1991; 126(2): 127-30.

22. Yoshimatsu H, Oomura $Y$, Katafuchi T, Niijima A. Effects of hypothalamic stimulation and lesion on adrenal nerve activity. Am J Physiol. 1987; 253(3 Pt 2):R418-24.

23. Plagemann A, Harder T, Rake A, Waas T, Melchior $K$, Ziska $T$, et al. Observations on the orexigenic hypothalamic neuropeptide Y-system in neonatally overfed weanling rats. J Neuroendocrinol. 1999; 11(7):541-6.

24. Torrezan R, Rodrigo MG, Ferrarese $M$, Melo FB Ramos AMD, Mathias PCF, et al. Treatmet with isoflavones replaces estradiol effect on tissue fat accumulation from ovariectomized rats. Arq Bras Endocrinol Metabol. 2008; 52(9):1489-96.

25. Leon-Quinto T, Magnan C, Portha B. Altered activity of the autonomous nervous system as a determinant of the impaired beta-cell secretory response after protein-energy restriction in the rat. Endocrinology. 1998; 139(8):3382-9.

26. Bernardis LL, Patterson BD. Correlation between "Lee Index" and carcass fat content in weanling and adults female rats with hypothalamic lesions. J Endocrinol. 1968; 40(4):527-28.

27. Plagemann A. Perinatal programming and functional teratogenesis: impact on body weight regulation and obesity. Physiol Behav. 2005; 86(5): 661-8.

28. Plagemann A, Heidrich I, Gotz F, Rohde W, Dorner G. Obesity and enhanced diabetes and cardiovascular risk in adult rats due to early postnatal overfeeding. Exp Clin Endocrinol. 1992: 99(3):154-8.

29. Elfers C, Ralston M, Roth CL. Studies of different female rat models of hypothalamic obesity. J Pediatr Endocrinol Metab. 2011; 24(3-4):131-7.

30. Amaral AG, Rafacho A, Oliveira CAM, Batista TM, Ribeiro RA, Latorraca MQ, et al. Leucine supplementation augments insulin secretion in pancreatic islets of malnourished mice. Pancreas. 2010; 39(6):847-55.

31. Harder T, Rake A, Rohde W, Doerner G, Plagemann A. Overweight and increased diabetes susceptibility in neonatally insulin-treated adult rats. Endocr Regul. 1999; 33(1):25-31.

32. Nascimento AF, Sugizaki MM, Leopoldo AS, LimaLeopoldo AP, Luvizotto RA, Nogueira CR, et al. A hypercaloric pellet-diet cycle induces obesity and co-morbidities in Wistar rats. Arq Bras Endocrinol Metabol. 2008; 52(6):968-74.

33. Hahn P. Effect of litter size on plasma cholesterol and insulin and some liver and adipose tissue enzymes in adult rodents. J Nutr. 1984; 114(7): 1231-4.

34. Mitrani P, Srinivasan M, Dodds C, Patel MS. Autonomic involvement in the permanent metabolic programming of hyperinsulinemia in the high-carbohydrate rat model. Am J Physiol Endocrinol Metab. 2007; 292(5):E1364-77.

35. Martins AGB, Branco RCS, Candido IC, Dias MJ, Rinaldi W, de Oliveira JC, et al. Dependência da atividade no controle glicêmico de ratos. Endocrinol Diabetes Clin Experim. 2008; 8(4):906-10.

36. Scheurink AJ, Steffens AB, Gaykema RP. Hypothalamic adrenoceptors mediate sympathoadrenal activity in exercising rats. Am J Physiol. 1990; 259(3 Pt 2): R470-7.

37. Kubera B, Hubold C, Zug S, Wischnath H, Wilhelm I, Hallschmid M, et al. The brain's supply and demand in obesity. Front Neuroenergetics. 2012; 4:4.

38. Balbo SL, Grassiolli S, Ribeiro RA, Bonfleur ML, Gravena C, Brito MN, et al. Fat storage is partially dependent on vagal activity and insulin secretion of hypothalamic obese rat. Endocrine. 2007; 31(2): 142-8.

39. Patterson CM, Levin BE. Role of exercise in the central regulation of energy homeostasis and in the prevention of obesity. Neuroendocrinology. 2008; 87(2):65-70.

40. Lausier J, Diaz WC, Roskens V, LaRock K, Herzer K, Fong CG, et al. Vagal control of pancreatic ss-cell proliferation. Am J Physiol Endocrinol Metab. 2010; 299(5):E786-93.

Recebido em: 28/9/2011 Versão final em: 20/3/2012 Aprovado em: 16/5/2012 